\title{
LA PROYECCIÓN SOCIAL EN LA UNIVERSIDAD DEL SIGLO XXI: IMAGINANDO ESPACIOS POLÍTICOS FUERA DEL AULA DE CLASE*
}

THE SOCIAL PROJECTION IN THE 21ST CENTURY UNIVERSITY:
IMAGINING POLITICAL SPACES OUTSIDE THE CLASSROOM

\author{
ANGie LoRena Fontecha, Pilar López RUIZ** \\ MónICA Gómez, LizetTe A. Mendoza y ÉdgaR GIovanNi Rodríguez***
}

Recibido: 20 de agosto de 2017 - Aceptado: 20 de septiembre de 2017 - Publicado 31 julio de 2018 DOI: $10.24142 /$ raju.v13n26a1

\section{Resumen}

Diversos estudios problematizan la relación Universidad-Sociedad, la cual debería ser imaginada como una relación dialógica y constructiva, tal como lo plantea Boaventura de Sousa (2012); por el contrario, la época actual establece diversas tensiones para la concepción de una universidad que se aleja de la interacción con la condición popular, cada vez más capturada por las lógicas del capital y la agenda públi-

Este artículo es resultado del proceso de investigación formativa en el semillero Bilingüismo e Interculturalidad de la Escuela de Educación, Fundación Universitaria Unimonserrate, Bogotá.

** Estudiantes de Licenciatura en Educación Bilingüe, Escuela de Educación, Fundación Universitaria Unimonserrate, Bogotá.

*** Profesores Escuela de Educación, Fundación Universitaria Unimonserrate, Bogotá. Correos electrónicos: profesionalescuelae@unimonserrate.edu.co, direccionbilingue@unimonserrate.edu.co y coordinacioninvestigacione@unimonserrate.edu.co 
ca de las agencias de cooperación y el interés trasnacional. En esta perspectiva, el siguiente texto explora la potencia de un experimento pedagógico que propone otro marco de sentido para la proyección social, mediante estrategias que promuevan la formación de maestros que, desde sus prácticas, movilicen el espacio público, el patrimonio, la ciudad y sus interacciones culturales como lugar de encuentro pedagógico y de producción de subjetividades — otras— más allá de los espacios convencionales de lo "escolar", en relación con la apertura al bilingüismo como pretexto de movilización y agencia de lo político.

Palabras clave: Educación popular, proyección social universitaria, educación bilingüe, pedagogías expandidas.

\section{Abstract}

Various studies problematize the University-Society relationship, which, should be imagined as a dialogical and constructive relationship as proposed by Boaventura de Sousa Santos (2012), on the contrary, the current time establishes various tensions for the conception of a university that moves away from the interaction with the popular condition, increasingly captured by the logic of capital and the public agenda of the cooperating agencies and the transnational interest. In this perspective, the following text explores the power of a pedagogical experiment that proposes another framework of meaning for social projection, through strategies that promote teacher training whom, from their practices, mobilize public space, heritage, the city and its cultural interactions as a place of pedagogical encounter and production of subjectivities -others- beyond the conventional spaces of the "scholastic", in relation to the opening to bilingualism as a pretext of mobilization and agency of the political.

Keywords: Popular education, university's social projection, bilingual education, expanded pedagogies. 
La proyección social en la universidad del siglo XXI:

imaginando espacios políticos fuera del aula de clase

\section{INTRODUCCIÓN}

Uno de los focos de atención frecuente en las políticas públicas en educación está relacionado con el logro de una educación bilingüe. Para las universidades y centros de educación superior el asunto ha significado acalorados debates en los cuales se enfrentan visiones que, atendiendo a una vocación epistémica cercana a los discursos decoloniales, defienden una multiculturalidad y el reconocimiento de los lenguajes originarios. Por otro lado, existe una visión del bilingüismo que reduce el asunto a un plano más funcional y que, desde una tradición moderna, establece relaciones directas entre el éxito profesional y la adquisición de una segunda lengua como garantía de un desempeño adecuado.

El paradigma del cual se parte supone un mayor rendimiento escolar y la adquisición de habilidades y competencias que estén más cercanas con los indicadores internacionales favorables a los procesos de inversión y competitividad. Una educación de alta calidad y una visión pragmática y funcional, derivada de este principio, implica un perfil de estudiantes con capacidades de alto rendimiento en el pensamiento lógico matemático y una habilidad en una segunda lengua para difundir sus ideas; o por lo menos para comprender directivas ejecutivas, todo ello en el marco de un modelo de "nivel de vida" y "progreso". Así:

El desarrollo socioeconómico de las naciones modernas viene acompañado de políticas e iniciativas orientadas hacia el mejoramiento de la calidad de vida de sus habitantes, mediante la implementación de diversos proyectos en educación, salud y seguridad, entre otros. Uno de los aspectos que resaltan los gobiernos a nivel mundial para el mejoramiento de las condiciones de bienestar de sus pobladores es su desarrollo profesional; es decir, las oportunidades brindadas a la población para su crecimiento académico y laboral (Bonilla y Rojas, 2012, p. 1).

La preminencia del idioma inglés establece no solo una manera particular de usar un sistema de codificación idiomática, sino sobre una hegemonía cultural, una ontología compartida que hace énfasis en los imaginarios del american lifestyle. Hablar una lengua extranjera, bajo las condiciones de nuestra sociedad es, en ocasiones, sinónimo de arribismo o de posibilidades que son aprovechadas solamente por una minoría... aquella que tiene las 
condiciones reales para la expresión de dicho poder. De fondo, no se trata de una cuestión baladí, sino de un saber-poder que determina, en este mismo sistema, unas ventajas competitivas.

Si bien es cierto, se considera que esta atención al idioma inglés resulta conexa a los fenómenos de globalización, financiarización e inversión extranjera, es también palpable que existe una dificultad en el aprendizaje de dicho idioma a pesar de que la cultura favorece dicho modelo. Esto resulta paradójico si se comparan las tasas o porcentajes de las evaluaciones en la competencia del idioma y los referentes culturales más usuales que son puramente anglos. En este orden de ideas, la proyección social universitaria corre el mismo riesgo, bajo el parámetro de la inversión y el logro económico se descuida o se desvanece su sentido dentro de la lógica universitaria que es, precisamente, impactar, de forma afirmativa, la relación academia-sociedad, teoría y práctica. Por ello, el fenómeno de la educación bilingüe y la proyección social se juntan como expresiones de un mismo síntoma: la universidad pensada para suplir el mercado y no la formación y subjetividades de sus miembros.

El experimento pedagógico que compartimos en este documento intenta mostrar de qué manera una estrategia de secuenciación didáctica, a la luz de un escenario de práctica y un curso en particular (Gestión educativa) para estudiantes del programa de licenciatura en educación bilingüe, puede aportar, significativamente, para incorporar en la vida universitaria habitual el sentido de la proyección social y comprender que la misma es la expresión vital de una comunidad que es capaz de efectuar sus valores en contextos distintos. Por ello, la gestión educativa, en relación con la proyección social en un escenario bilingüe, no es otra cosa que un pretexto para problematizar, identificar y evaluar hasta dónde la implementación de dichas transformaciones en la enseñanza habitual del idioma extranjero puede o no suscitar reflexiones más profundas. ¿Puede ser la enseñanza del idioma extranjero un vehículo de extensión y proyección social? ¿Políticamente, qué genera en los maestros en formación? ¿Cómo altera la dinámica de planeación de prácticas y de sistematización de experiencias? ¿Qué se puede aprender de ello?

\section{LA PROYECCIÓN SOCIAL Y EXTENSIÓN EN LAS UNIVERSIDADES LATINOAMERICANAS: HISTORIA Y TENSIONES}

Morales y Ortiz (2011) ubican el origen del concepto de extensión en las universidades latinoamericanas como consecuencia del movimiento o 
La proyección social en la universidad del siglo XXI:

imaginando espacios políticos fuera del aula de clase

la Reforma Universitaria de Córdoba, iniciada en 1918 en Argentina y con repercusión en toda la región. Tünnermann (2008) la define como "el primer cuestionamiento serio de la Universidad Latinoamericana tradicional" (p. 1), una universidad diseñada para la reproducción de la cultura y el conocimiento y exclusiva de las clases dominantes. Este movimiento abrió la discusión, aún vigente, sobre la democratización de las universidades y, con ello, la vinculación de la extensión universitaria y la difusión cultural. Es de este modo como Morales y Ortiz (2011), citando a Tünnermann, señalan:

De esta suerte, el movimiento agregó al tríptico misional clásico de la Universidad un nuevo y prometedor cometido, capaz de vincularla más estrechamente con la sociedad y sus problemas, de volcarla hacia su pueblo, haciendo a este partícipe de su mensaje y transformándose en su conciencia cívica y social. Acorde con esta aspiración, la Reforma incorporó la extensión universitaria y la difusión cultural entre las tareas normales de la Universidad latinoamericana y propugnó por hacer de ella el centro por excelencia para el estudio científico y objetivo de los grandes problemas nacionales (p. 3).

Medio siglo más tarde, en la II Conferencia Latinoamericana de Difusión Cultural y Extensión Universitaria, llevada a cabo en México en febrero de 1978, se problematizó alrededor de qué se debía entender por extensión, la vinculación de los estudiantes en ella y el deber ser de las universidades latinoamericanas, postulando así la siguiente definición de extensión universitaria:

es la interacción entre la universidad y los demás componentes del cuerpo social, a través de la cual esta asume y cumple su compromiso de participación en el proceso social de creación de la cultura y de la liberación y transformación radical de la comunidad nacional (Morales y Ortiz, 2011, p. 1).

Sin embargo, aunque en América Latina existan algunos escenarios de discusión, en los cuales cobra importancia y relevancia la extensión universitaria como parte fundamental para la transformación del engranaje social, político y cultural de una nación, el contexto global ha llevado a las universidades a la multiversidad; es decir, la universidad y la extensión con fines instrumentales y funcionales para el mantenimiento del orden hegemónico. Es por esta razón que Serna (2007) describe tres modelos de extensión 
que promueven la multiversidad: el Modelo de Vinculación Empresarial, que es el diálogo entre empresa-universidad o agencias de cooperación-universidad, el cual limita las necesidades de una nación a los requerimientos del sector productivo y económico. El Modelo Altruista, basado en "las acciones desinteresadas de los universitarios en favor de las poblaciones marginadas. De esta visión surgen las brigadas de salud, los consultorios jurídicos, la capacitación a poblaciones vulnerables y las pasantías sociales de los estudiantes" (Morales y Ortiz, 2011, p. 4) y, por último, el Modelo Divulgativo, que nace con la intención de elevar el nivel cultural de la población y no está sujeto a las necesidades de la misma; este modelo, además, considera que el problema de apropiación del conocimiento es "solo de tipo técnico o comunicativo, se erige como otro instrumento del proyecto modernizador que otorga la mayor $-\mathrm{y}$ a veces la única-, validez a las expresiones culturales o tecnológicas producidas por artistas e intelectuales de instituciones extranjeras" (Serna, 2007, p. 3).

Frente a esta visión funcionalista de la universidad, Boaventura de Sousa (2012) describe cómo la multiversidad impide la articulación entre la docencia, la investigación y la proyección social, volviéndolas contradictorias y creándole a la universidad las siguientes funciones:

educación general postsecundaria, investigación, suministro de mano de obra calificada, educación y entrenamiento altamente especializados, fortalecimiento de la competitividad de la economía, mecanismos de selección para empleo de alto nivel, movilidad social para hijos e hijas de las familias proletarias, prestación de servicios a la región y a la comunidad local, paradigmas de aplicación de políticas nacionales, preparación para los papeles de liderazgo social, entre otras (p. 227).

Cabe señalar que Serna (2007) hace mención a un último modelo que rompe con la visión funcionalista de la universidad; este surge de "las ideas [de] la izquierda latinoamericana y enriquecido con el pensamiento de Freire, que pondera compartir los bienes, incluidos los educativos y culturales, en forma dialógica y liberadora, creando conciencia entre participantes en ese acto educativo" (p. 3). Un ejemplo de este modelo lo trae Boaventura de Sousa al hablar de la experiencia de la Universidad de Brasilia, que intenta poner en valor los saberes y conocimientos populares y ancestrales, ya que: 
La proyección social en la universidad del siglo XXI:

imaginando espacios políticos fuera del aula de clase

se considera que el conocimiento científico, tecnológico y artístico generados en la universidad e institutos de investigación no son únicos. Existen otras formas de conocimiento surgidas de la manera de pensar y actuar de los innumerables segmentos de la sociedad a lo largo de generaciones que, por no estar caracterizados como científicas, están desprovistas de legitimidad institucional (De Sousa, 2012, p. 254).

Es por lo ya dicho que, a continuación, se pretende reflexionar en torno a una proyección social nacida desde el diálogo y para la transformación.

\section{APUESTAS DE LA PROYECCIÓN SOCIAL DESDE EL DIÁLOGO PARA LA TRANSFORMACIÓN}

O la Extensión vive ahí en eso que hacen los estudiantes o poco servirá para transformar alguna cosa en la Universidad

Tommasino (2015, p. 43)

Una de las preguntas que debería estar en permanente discusión en las universidades es ¿cómo hacer una proyección social dialógica y transformadora? Para lo anterior es necesario pensar, además, ¿cómo volver una institución conservadora y hegemónica en una instituyente y autónoma? Pareciera que estas preguntas se resolverían si las universidades articularan entre sí las funciones sustantivas (docencia, investigación y extensión); sin embargo, es necesario pensar la participación de los estudiantes y docentes en la extensión, y esto implica concebir la investigación y la docencia fuera del aula de clase, pensar que las instituciones y lo cotidiano también forman parte del acto educativo e implica concebir una universidad abierta "sin muros", ya que como señala Tommasino (2015):

La gran mayoría del tiempo institucional es docente-estudiantil. Esta conceptualización de Extensión, imbricada en el acto educativo, jaquea la pedagogía que se instaló históricamente en las Universidades Públicas. Esta incorporación de la Extensión en la currícula normal también pone en jaque a la "educación bancaria" que, en general, tenemos con nuestros estudiantes en las aulas. Esta concepción de Extensión, imbricada en el acto educativo, jaquea la posibilidad de "iluminar al estudiante", y no digo que la destruye, pero sí que la condiciona (p. 50). 
De este modo, la universidad tiene la obligación de resignificar la extensión, viéndola como un campo problemático que establece relaciones con otros campos y pone en diálogo el saber académico y el popular. Es así como la extensión tiene múltiples apuestas, desde lo pedagógico, lo político y lo epistémico (Cano, 2014).

En lo pedagógico es importante pensar la necesidad o no de incorporar la extensión universitaria al currículo, ya que según Cano (2014) esto permitiría constituir la extensión como un proceso educativo que incorpora a los estudiantes, docentes y actores sociales fuera de la universidad. En cuanto a lo político, hay que tener presente que la extensión universitaria condensa los conflictos propios de un proceso de institución y su objetivo debe ser buscar y abrir "nuevos modos de articulación entre la universidad y la sociedad" (Cano, 2014, p. 49) con un fin transformador y emancipatorio. Y, por último, lo ético-metodológico debe pensarse como una apuesta transversal a lo pedagógico y lo político, ya que implica la superación de la visión tradicional de la extensión como asistencialismo social y cultural y, por el contrario, invita a pensar en otras maneras de articular conocimientos, prácticas, acciones y sujetos.

Es de esta manera como la extensión cobra otro sentido y se convierte en un proceso de interrogación, diálogo y creación de conocimientos donde el "saber científico pueda dialogar con el saber laico, con el saber popular, con el saber de los indígenas, con el saber de las poblaciones urbanas marginales, con el saber campesino" (De Sousa, 2006, p. 26). Es así como para Unimonserrate la extensión se constituye en

una práctica académica y social cuya intención es establecer un diálogo entre los saberes especializados de la academia y los saberes o experiencias sociales de otros grupos, posibilitando una interacción activa entre la IES y organizaciones sociales y culturales, o del sector productivo, entre otros (Unimonserrate - Fundación Universitaria Monserrate, 2005, p. 38).

Esta naturaleza dialógica de la proyección social implica que Unimonserrate asuma a los agentes sociales como interlocutores válidos, de quienes se puede aprender y con quienes vale la pena crecer institucionalmente. 
La proyección social en la universidad del siglo XXI:

imaginando espacios políticos fuera del aula de clase

\section{UN HORIZONTE PEDAGÓGICO PARA LA EDUCACIÓN BILINGÜE}

Genesee (2004) define la educación bilingüe como el tipo de educación cuyo objetivo es promocionar la suficiencia bilingüe, usando ambas lenguas como medios de enseñanza para porciones significantes del currículo académico; definiendo esta suficiencia como la habilidad de usar la lengua meta de manera efectiva y apropiada para auténticos propósitos personales, educativos, sociales o relacionados con el trabajo.

En la Licenciatura en Educación Bilingüe de la Escuela de Educación, los profesores en formación están expuestos a un programa intensivo de inglés, y una vez consolidadas las bases estudian varias de los cursos de contenido utilizando el inglés como lengua de enseñanza. Esta formación va acompañada del desarrollo de habilidades que les permite tener un perfil como gestores de cultura, donde aprenden y enseñan teniendo en cuenta los contenidos, la comunicación, la cognición y la cultura, sin ir en detrimento de la primera lengua, a la cual se le hace especial énfasis por tratarse de educación bilingüe y se refuerza su buen uso y conocimiento.

Para la formación de profesores de la Licenciatura en Educación Bilingüe de la Escuela de Educación es esencial promover un bilingüismo funcional y aditivo, que responda a las necesidades cognitivas individuales y sociales de quien lo desarrolle, sin detrimento ni de la cultura colombiana ni del español como lengua materna.

En este sentido, se ha buscado que los espacios de formación de los estudiantes sean diseñados como ambientes de aprendizaje que fomenten el bilingüismo funcional aditivo; es decir, que el enfoque de enseñanza de la lengua materna y la extranjera se oriente hacia su uso funcional, y que el aprendizaje de la segunda lengua enriquezca el aprendizaje de la materna y viceversa (Lambert, 1975).

De igual manera, se considera que para el diseño de dichos ambientes de aprendizaje, donde se promueva el bilingüismo, se requiere, además del desarrollo de las cuatro habilidades comunicativas: habla, escucha, escritura y lectura, la ampliación de habilidades interculturales y la metacognición.

Los profesores de la Licenciatura, gracias a su formación bilingüe, desarrollan la función cognitiva del lenguaje, la cual utilizan para organizar y analizar el conocimiento, facilitando los procesos de autorregulación y de feedback a nivel metalingüístico y metacognitivo (Baker, 2006). En cuanto a sus habilidades interculturales, se fomenta el reconocimiento del pluralismo cultural o multiculturalismo, de manera que el futuro profesor licenciado en 
Educación Bilingüe sea capaz de reconocer el modelo de organización de las diferentes comunidades, de diferentes culturas, lenguas, religiones, etc., y cómo estas pueden vivir armónicamente. Para Argibay (2003), las personas deben dar valor positivo a la diversidad sociocultural y los profesores deben promover, desde la enseñanza, que los grupos o comunidades no pierdan su identidad y cultura propias.

Los espacios de formación que se han diseñado fuera del aula (como forma de proyección social y extensión de la vida universitaria) son Campamentos de Inmersión Lingüística, city tours y encuentros interculturales.

\section{LA PRÁCTICA DE GESTIÓN EDUCATIVA BILINGÜE Y LOS ESPACIOS DE FORMACIÓN A LOS OJOS DE SUS ESTUDIANTES}

Considerar los procesos de documentación pedagógica y de registro es fundamental para realizar evaluaciones situadas de las experiencias de práctica. Por esta razón, traemos algunas de las voces que emanan de los registros realizados por nuestras estudiantes, dado que ello nos permite visualizar varios aspectos:

- El nivel de interiorización de los sentidos y los propósitos, tanto del programa como de la apuesta particular en el curso de "gestión educativa".

- Las habilidades escriturales y comunicativas que, gradualmente, las estudiantes han formalizado y que les permite hablar de sus prácticas.

- Los aspectos apreciativos que revelan juicios de valor o percepciones que se decantan de manera diferencial.

Tal como lo enuncia Maturana (2011), a partir de una investigación previa la cuestión pedagógica del "éxito" en el proceso de formación como profesor depende de relaciones distintas (incluso más allá de la experticia o dominio técnico del idioma). Como se trata de un aprendizaje de base cultural parece que la calidad de las interacciones (trabajo en equipo y colaborativo, proxemia, etc.) son definitivas en el aprendizaje. 
La proyección social en la universidad del siglo XXI:

imaginando espacios políticos fuera del aula de clase

Lo anterior demuestra la estrecha relación entre las estrategias de enseñanza y el rol del maestro. A partir de los datos se infiere que en la interacción maestra-estudiante el papel es de apoyo mutuo. A pesar de que la mayoría de las maestras de inglés no se sienten plenamente identificadas con este rol, sí promueven en sus aulas de clase un ambiente de cooperación, aprendizaje mutuo y de participación; elementos característicos de un aula de clase centrada en el estudiante. Aunque la falta de identificación con la enseñanza del inglés se debe en parte a que es una actividad a la que llegaron bien de manera incidental, por haber demostrado algún interés en la lengua o haber realizado algunos cursos; o bien de manera impuesta, como resultado de la dinámica administrativa de las instituciones donde laboran, todas ellas demuestran un gran sentido de ética profesional y de responsabilidad social con sus estudiantes (Cadavid et al., 2004, citado por Maturana, 2011, p. 85).

Veamos las percepciones derivadas de los diarios de campo:

La práctica de gestión educativa es un proceso de formación muy interesante, ya que nos ha permitido entender que hay otros espacios en los que podemos interactuar, aprender y conocer más allá de nuestros conocimientos previos. La gestión educativa la podríamos entender como un apoyo en el área educativa, todo lo que tiene que ver con eventos y actividades que realiza una institución o universidad.

El texto supone, de entrada, un cuestionamiento sobre la claridad que logran los estudiantes en relación con la noción de "gestión educativa". Si bien es significativo su compromiso para la planeación de actividades, llama mucho la atención que la gestión se relacione con la organización de eventos. Sin embargo, sí se establece una relación directa con otro tipo de aprendizajes, por lo que resulta ser un logro importante.

Para ello nosotros hemos hecho varias actividades que fortalecieron un aprendizaje en el que en esos espacios el contenido era una excusa para aprender el inglés. Nosotros redactamos nuestras experiencias de las actividades mediante un diario de campo en donde allí podíamos reflexionar acerca de la práctica; esta forma de exploración de la práctica creemos que permite esclarecer el rol que cumple la reflexión de ella en las relaciones teoría-práctica y apoya la construcción de algunas competencias necesarias en el proceso de formación. 
La práctica puede ayudarnos a identificar problemas y buscar estrategias para mejorar las problemáticas que hay en la educación; por lo tanto, es fundamental que los docentes en formación tengan, desde sus primeros semestres, prácticas para enfrentarse a la vida real de un maestro y de que es posible generar un cambio para un mejor aprendizaje para los estudiantes.

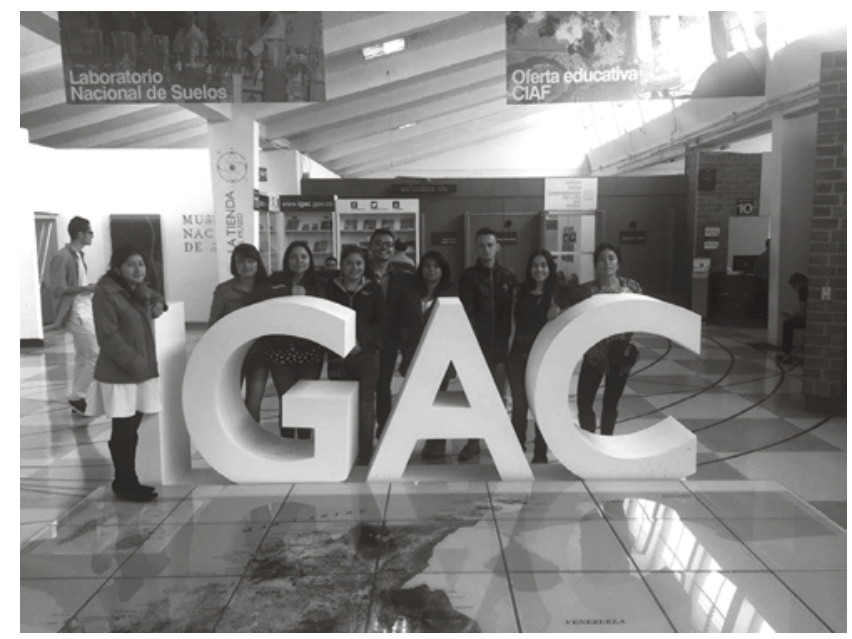

Figura 1.1 Visita al Instituto Geográfico Agustín Codazzi (IGAC).

Las competencias en el idioma inglés se aprenden en contextos de gestión cultural y de promoción y divulgación de la información científica de acuerdo con los énfasis del programa. La proyección social implica la visibilización y participación con otras comunidades académicas.

El diario de campo es una herramienta de registro, supone diferentes técnicas de captura de información, de compilación de datos y notas de experiencias pero, sobre todo, es una herramienta que facilita la praxis; es decir, un dispositivo para que el maestro pueda reconocerse a través de sus propios diseños de aprendizaje, escuchar sus propias voces y las de los participantes de sus intervenciones. En el texto se puede verificar que las estudiantes reconocen en la vivencia del proyecto una intencionalidad de la práctica (que va más allá del aprendizaje del idioma) y una noción que es cercana a la búsqueda de sentidos pedagógicos del programa y el profesor a cargo del curso. 
$\mathrm{El}$ encuentro intercultural es muy interesante porque aprendemos de las diferentes culturas e interactuamos con otras personas. Pudimos observar que en aquel espacio había frustraciones por parte de los estudiantes de la carrera de preescolar, ya que fueron los únicos (incluyendo Educación Bilingüe) que estuvieron en el encuentro. Los estudiantes no podían comprender lo que los invitados querían expresar. En esta primera actividad no tuvimos mucha participación, ya que aún no sabíamos cómo iban a ser nuestras prácticas.

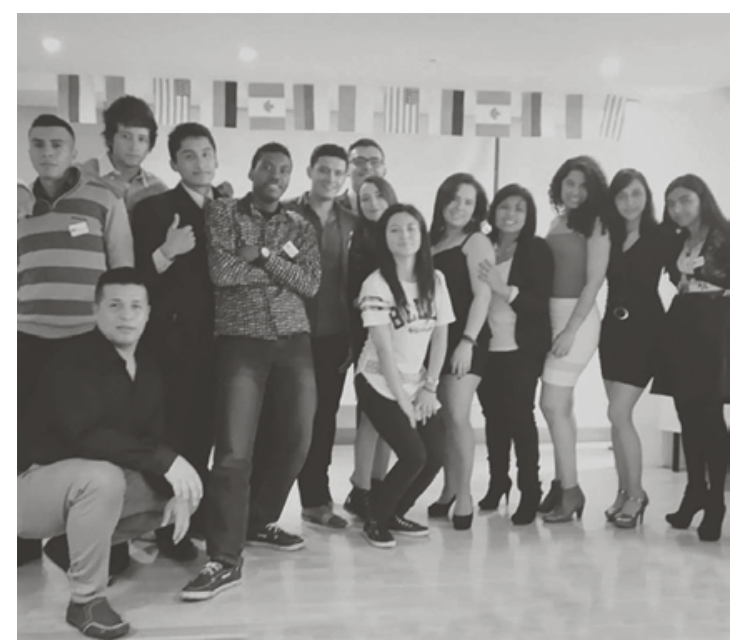

Figura 1.2 Detalle del encuentro intercultural.

No solo se ponen en juego las comprensiones del lenguaje, sino las habilidades y competencias sociales adecuadas para promover un diálogo respetuoso de la diferencia.

Si bien es cierto que dentro de la estrategia se proponen diferentes espacios, pareciera que para los estudiantes existen momentos de ausencia de claridad, por más que el profesor a cargo realice diferentes intervenciones para clarificar su propósito. Es interesante, en la sección del párrafo, de qué manera se percibe la importancia de la participación cuando se inicia afirmando que uno de los aspectos principales es la "interacción con otras personas"; esto denota que, en efecto, existe un riesgo en la generación de actividades y es precisamente que bajo las condiciones específicas se puede 
perder, con facilidad, el hilo conductor. Aquí es necesario resaltar que la interculturalidad puede ser una excusa para movilizar diferentes acciones de proyección social y de politización de quienes participan; pero el diálogo, el debate de las posiciones, de los hábitos, de las costumbres por contraste invita a que en realidad estemos dispuestos a aprender de otras culturas.

La segunda actividad fue un campamento dirigido por parte de Bilingüe donde_todo era "inmersión". Todas las actividades las lideraban algunos estudiantes de Bilinguie y el director del programa de Educación Bilingüe. En este espacio, desde que nos subíamos en los buses, se usaba el inglés, aprendiendo canciones en inglés para motivar a los estudiantes, para armar las carpas también se usaba el inglés y haciendo que los estudiantes al final repitieran las instrucciones.

El Campamento de inmersión es una de las actividades que tiene mayor acogida, pues implica una serie de representaciones sociales en las cuales los estudiantes se ven abocados como, por ejemplo, que se trata de la primera vez que salen sin sus padres y, en este sentido, comienzan a ser responsables de sus propios comportamientos. Estas novedades se resaltan con el comentario "todo es inmersión" pues, en realidad, no se trata solamente de la inmersión lingüística, sino en la práctica social misma y lo que produce en sus subjetividades. Distintos valores, percepciones, prejuicios se ponen en juego y esta serie de simbolizaciones y significaciones son las que ayudan a poblar la correlación entre el objetivo del campamento y los modos de lenguajear en contexto. Repetir la instrucción puede hacer parte de un proceso complejo que debe ser analizado en profundidad, pues el peso "de lo que se aprende" en la inmersión podría verse afectado por una instrumentalización de un recurso específico del idioma... algo que puedo replicar cuando sea necesario, sin situarlo, sin problematizarlo.

La tercera actividad que se realizó fue un city tour (en todos los semestres se realizan) por todo el eje ambiental (centro de Bogotá). Fue una planeación de aproximadamente un mes para escoger lugares y consultar todo acerca de su historia en inglés y que fuera una manera fácil de entender para que los estudiantes no se confundieran. Es muy importante conocer la historia de nuestro país, ya que hay muchas cosas que no sabemos de la sociedad; además, porque pasaron muchas cosas que hoy en día tenemos y no sabemos por qué están. Comen- 
zábamos a hacer preguntas sencillas como: What kind of place is it? si era en un lugar, y si eran monumentos de las personas que representan un papel importante en el país preguntamos: Who is he/she? y Why is he/she important in our country? Con estas preguntas ellos tenían que pensar en algo sencillo para responder usando el inglés. Esta experiencia nos da una muestra de que nosotros, como maestros en formación, podemos hacer uso de nuestra ciudad para poder enseñar el idioma y otras cosas como historia, que es un ejemplo de ello. Los estudiantes podían defenderse con frases muy simples o responder con verbos en inglés. Es difícil estar en frente de muchas personas que comprendían más el inglés que otros y que quizás lo criticaran a uno por ciertos errores que comúnmente uno comente cuando está en proceso de aprender una lengua.
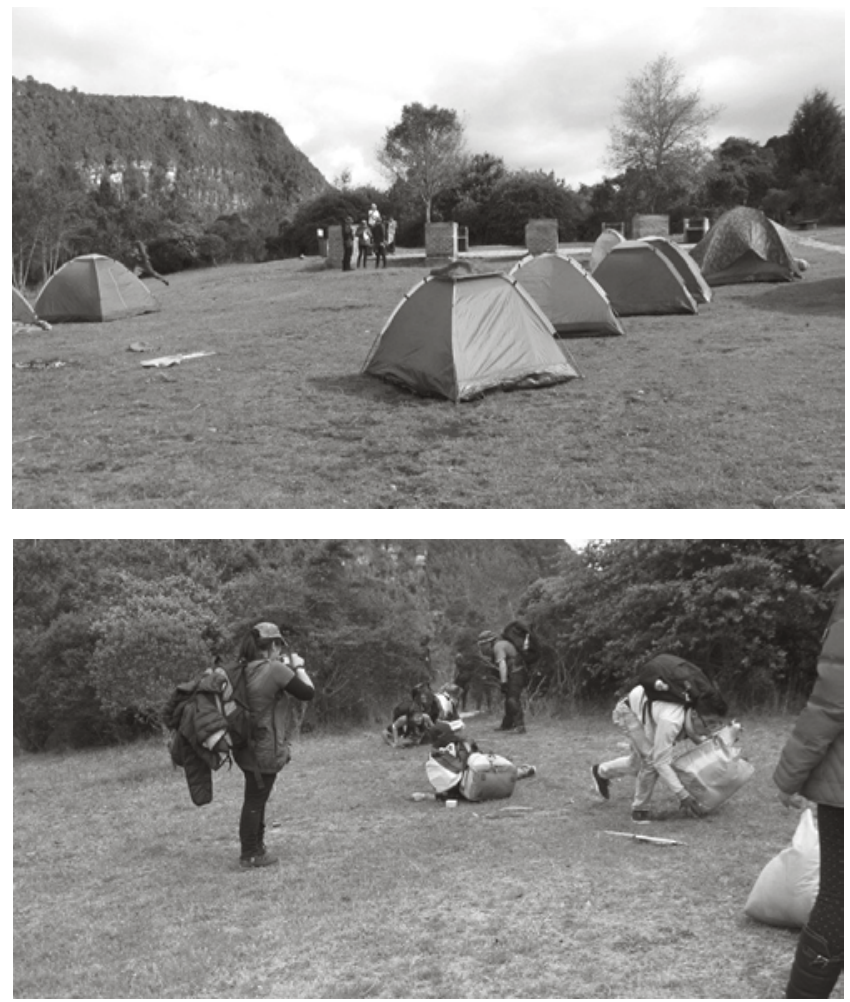

Figura 1.3 Campamento de inmersión. 


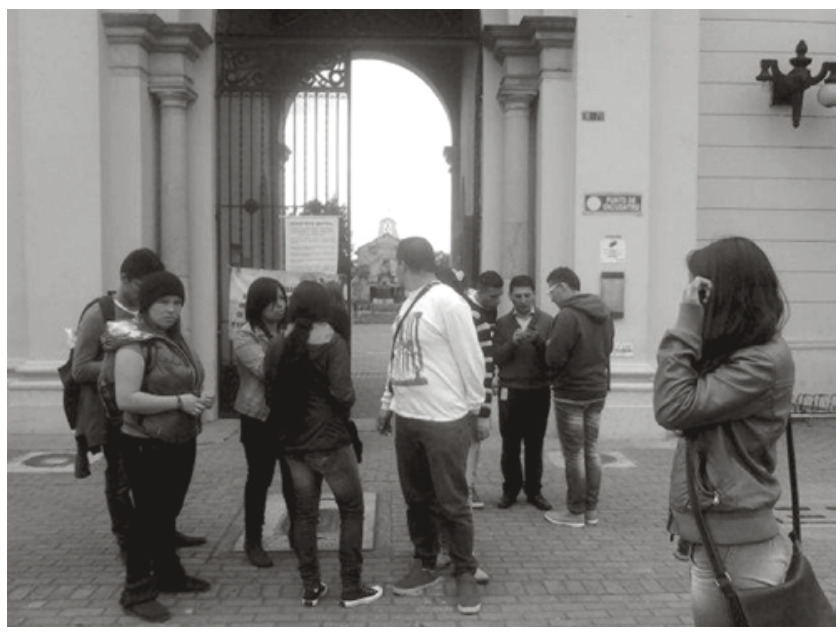

Figura 1.4 Visita al Cementerio Central de Bogotá.

Los espacios históricos en el contexto del city tour promueven el reconocimiento del patrimonio y de la historia. Con estos saberes se enfatiza el aprendizaje del idioma en contexto. En la fotografía, los profesores Daniel Hernández y Lizette Mendoza junto a sus estudiantes del Programa de Licenciatura en Educación Bilingüe.

Las actividades que se relacionan con salidas a terreno son muy importantes; se reconoce que este tipo de recurso favorece la implicación del estudiante y la importancia de su función dentro de la sociedad como ciudadano y como maestro en formación. La aprobación sigue siendo vital y condiciona el ejercicio en cierto sentido. La selección de lugares no clarifica la relación con la función de proyección social, lo que sería muy importante para fines de nuestra investigación. En este sentido, este primer trabajo abre muchas posibilidades para ajustar el proceso, pero también para encontrar mecanismos de interacción entre las funciones sustantivas, de lo cual, este primer ejercicio es un buen ejemplo.

Enfrentarnos a este tipo de prácticas fuera del aula nos ayudó a despertar algunas habilidades que quizás en un colegio no se hubieran podido despertar, como el poder transmitir de forma más creativa y más segura los conocimientos que queremos transmitirle a los estudiantes; que no solo el maestro puede estar enseñando inglés en un 
La proyección social en la universidad del siglo XXI:

imaginando espacios políticos fuera del aula de clase

salón de clase sino que se deben aprovechar todos los espacios que la ciudad nos ofrece. El aprender estas nuevas formas de enseñanza, saliéndose de la monotonía, nos ha enriquecido abriéndonos paso a un nuevo mundo donde el individuo aprende de distinta manera y nosotros debemos descubrir todas aquellas maneras para que no todos los estudiantes caigan en aprender lo mismo, sino en aprender siempre diferentes cosas. También hemos aprendido que, gracias a esta experiencia, vemos el mundo de otra manera, que podemos usar todo lo que nos rodea para aprender otro idioma y que la tecnología será una buena herramienta para sacarle provecho y que los estudiantes sientan que están en el siglo XXI y no en el XVI.

Sin duda, este último extracto de los diarios de campo es el más interesante, pues por un lado resalta la importancia de unas metodologías distintas en el escenario general de la educación, menciona el papel de la tecnología y destaca que es necesario salir de lo "monótono". Bajo estas claves discursivas es posible comprender que en el caso del programa y el efecto de estas secuencias didácticas, en el curso de gestión, existen unos reconocimientos sobre la capacidad de la innovación, pero aún hace falta recorrer el camino de la caracterización profunda de aquello que se aprende más allá del disfrute de lo novedoso. Se hace mención a un tipo de conocimiento, pero este no se identifica y no se logra establecer que queda derivado de la experiencia.

\section{CONCLUSIONES}

Tal como afirman Bonilla y Rojas (2012):

El aprendizaje del inglés como medio para salir de la pobreza es señalado por Córdoba (2011) como "el resultado del modelo económico escogido [el cual] ha propiciado la construcción de una identidad socio-educativa en la enseñanza y aprendizaje del inglés basada en la noción de empleo y, por ende, en la asociación metonímica con el estado económico de las personas" (p. 9). Esta postura demuestra la estrecha relación entre el aprendizaje de este idioma y la consecución de mejores opciones laborales, desde una perspectiva de desarrollo socioeconómico basada en la generación de empleo por parte de la empresa nacional y transnacional, las cuales requieren de personal adecuado a sus necesidades de mercado (p. 8). 
En la actualidad, la enseñanza de una segunda lengua, de una educación bilingüe, de la experticia en la apropiación de una cultura a través del lenguaje, supone preguntas inquietantes más que grandes certezas. Este breve ejercicio de investigación, sobre una práctica particular, nos permitió identificar elementos problemáticos que transitan como imaginarios, tanto en nuestros estudiantes como en nuestros docentes. Por un lado, apostamos a un posicionamiento de la proyección social como función trasversal de la universidad, que debe dar sentido a factores curriculares como investigativos. En esta mutua dependencia, sabemos que la proyección y la extensión se logran mediante nuevas estrategias que permitan a los estudiantes y profesores visibilizar en las comunidades el quehacer de lo universitario, más allá de una pretendida dominancia de saber-poder, se trata, como se vivió en este ejercicio, de experimentos didácticos que permitan aprendizajes compartidos, que motiven a los participantes a problematizar su identidad, su historia y su provenir. Extender socialmente y proyectar socialmente no es otra cosa que intentar devolver un espacio político perdido, o por lo menos refundido en las lógicas de calidad y de mercado: lograr una vivencia común, devenir comunidad, diseñar otros mapas y recorridos...

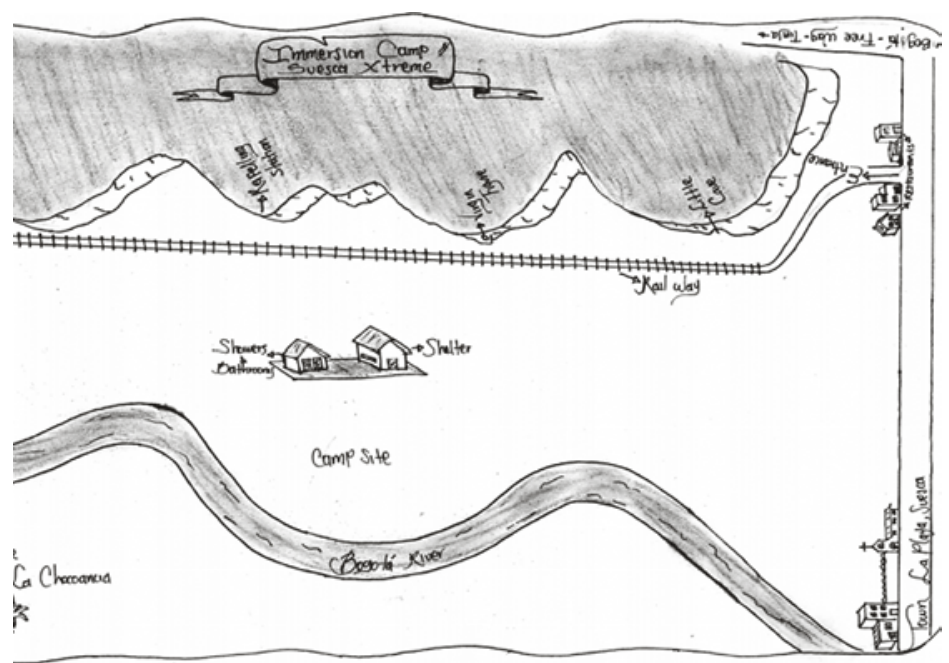

Figura 1.5 Proceso de cartografía realizado por estudiantes de Licenciatura en Educación Bilingüe como parte de su planeación de gestión educativa para la salida de inmersión a Suesca, Cundinamarca. 
La proyección social en la universidad del siglo XXI:

imaginando espacios políticos fuera del aula de clase

\section{REFERENCIAS}

Argibay, M (2003). Conceptos básicos. Recuperado de http://www. bantaba.ehu.es/formarse/ficheros/view/Exposici\%F3n_2_Sesi\%F3n_1.pdf?revision_id=34450\&package_id $=34415$

Baker, C. (2006). Foundations of Bilingual Education and Bilingualism. Clevedon: Multilingual Matters.

Bonilla, A., y Rojas, R. (2012). El aprendizaje de lenguas extranjeras como herramienta para el desarrollo humano [Memorias Congreso Iberoamericano de las Lenguas en la Educación y en la Cultura / IV Congreso Leer.es]. Salamanca, España.

Cano, J. (2014). La extensión universitaria en la transformación de la universidad latinoamericana del siglo XXI: disputas y desafíos. Recuperado de http://biblioteca.clacso.edu.ar/clacso/becas/20141202093928/ensayo_cano_premio_pedro_krotsch.pdf

De Mejía, A. (1998). Educación bilingüe en Colombia en contextos lingüísticos mayoritarios: hacia una caracterización del campo. Recuperado de http://bibliotecadigital.univalle.edu.co/bitstream/10893/5543/1/EDUCACION\%20BILINGUE.pdf

De Sousa, B. (2006). La Sociología de las Ausencias y la Sociología de las Emergencias: para una ecología de saberes. En Renovar la teoría crítica y reinventar la emanciapción social (encuentros en Buenos Aires). Buenos Aires: CLACSO. Recuperado de http://biblioteca.clacso.edu.ar/clacso/ coediciones/20100825033033/2CapituloI.pdf

De Sousa, B. (2012). De la mano de Alicia. Lo social y lo político en la postmodernidad. Bogotá: Siglo del Hombre.

Genesee, F. (2004). What do we Know About Bilingual Education for Majority Language Students? En T. K. Bhaktia \& W. Richie (Eds.), Handbook of Bilingualism and Multilingualism. Malden: Blackwell.

Lambert, W. E. (1975). Culture and Language as Factors in Learning and Education. En A. Wolfgang (Ed.), Education of Immigrant Students (pags. 55-83). Toronto: OISE Press.

Maturana, A. (2011). La enseñanza del inglés en tiempos del Plan Nacional de Bilingüismo en algunas instituciones públicas: factores lingüísticos y pedagógicos. Recuperado de http://www.scielo.org.co/pdf/calj/v13n2/ v13n2a06.pdf 
Morales, E., y Ortiz, C. (2011). La extensión universitaria en América Latina: concepciones y tendencias. Recuperado de http://educacionyeducadores.unisabana.edu.co/index.php/eye/article/view/1928/2515

Richards, C. J. (2006). Communicative Language Teaching Today. Nueva York: SEAMEO. Recuperado de https://www.researchgate.net/profile/Jack_Richards4/publication/242720833_Communicative_Language_ Teaching_Today/links/5580c02808aea3d7096e4ddb.pdf

II Conferencia Latinoamericana de Difusión Cultural y Extensión Universitaria (s. f.). Recuperado de http://resu.anuies.mx/archives/revistas/ Revista2_S3A1ES.pdf

Serna, G. (2007). Misión social y modelos de extensión universitaria: del entusiasmo al desdén. Revista Iberoamericana de Educación, (43), 3-7.

Tommasino, H. (2015). El rol de los estudiantes en los procesos extensionistas. En J. M. Medina (Comp.), La Universidad en diálogo con la comunidad: construyendo una institución en contexto (págs. 43-51). Rosario: UNR Editora.

Tünnermann, C. (2008). La Reforma de Córdoba. Vientre fecundo de la transformación universitaria. En La reforma universitaria: desafios y perspectivas noventa años después (págs. 16-19). Buenos Aires: CLACSO. Recuperado de http://biblioteca.clacso.edu.ar/ar/libros/grupos/reforAboit/ 03tunn.pdf

Unimonserrate - Fundación Universitaria Monserrate (2005). Políticas institucionales. Consejo Superior. Acuerdo. 1-0120 del 6 de mayo de 2004. Bogotá [documento interno]. 